\title{
PAPR Reduction for FBMC Systems with Ant Bee Colony Optimization
}

\author{
V.Sudharshini kataksham ${ }^{1}$, Dr.P.Siddaiah ${ }^{2}$ \\ ${ }^{1}$ Research Scholar, Department of ECE, AN U college of Engg\& Technology, Nagarjuna University, AP, India \\ ${ }^{2}$ Professor, Department of ECE, AN U college of Engg\& Technology, Nagarjuna University, AP, India
}

\begin{abstract}
Multi carrier systems are most prevalent modern wireless communication systems which are employed for high data rate transmission. At present, FBMC (Filter bank multi carrier) with OQAM (Offset-QAM) has drawing attention for many researchers to implement new physical layers and wireless protocols. As in the case of multi carrier system, this FBMC also suffers from high PAPR, so this paper presents a PAPR reduction approach with ant bee colony (ABC) optimization. ABC algorithm functionality is similar to honey mining where the labor is shared for nectar source and hence find out the optimal solution. Experimental results shows that the proposed approach could able to attain considerable improvement in decreasing the PAPR when compared against the conventional FBMC and SLM (selective mapping method) based FBMC approaches.
\end{abstract}

Key words: FBMC, SLM, PAPR reduction, Ant bee Colony optimization

\section{INTRODUCTION}

Many improvements in the physical layer technologies were witnessed and their implementations in wireless communication system during the past decade. With this rapid growth and advancements in the wireless technologies and personal wireless equipment's the physical layer implementation may go beyond 5G in future days. OFDM (orthogonal frequency division multiplexing) provides high power efficiency of the physical layer apart from providing tolerance towards the multipath delay spread and frequency selective fading channels. In certain cases, like in IEEE 802.11a, this approach provides a high data rate of about $54 \mathrm{Mbps}$ operating in $20 \mathrm{MHz}$ channel [1]. There are few limitations with this approach as it is more open to phase noise and frequency offset. On other hand there is a decrease in the power efficiency of the system due to high PAPR, therefore these limitation has to be overcome if the approach has to be implemented in high data rate wireless communication systems.

Filter band Multi carrier (FBMC), reduces the out of band interference $(\mathrm{OBI})$ by selecting pulse shaping filter for each subcarrier so that multiple sub carriers can be accommodated in the limited frequency band. To achieve this, Saltzberg [2] proposed a method of shifting half of the In-phase (I) and Quadrature Phase (Q) components of IQ modulated symbols. This analysis makes it possible to achieve a high baud rate between the adjacent sub carriers and also can recover the information symbols there by reducing the impact of Inter symbol interference (ISI) and inter carrier interference (ICI) [3]. The major difference between OFDM and FBMC lies in spectral efficiency which is clearly depicted in Figure 1, it can be observed that the frequency response of the OFDM do possess side lobe leaking that reduces the orthogonality between the sub carriers. On other hand FBMC has negligible side leakage in the frequency response, with this property low spectral leakage low adjacent channel interference and high spectrum resolution can be achieved.

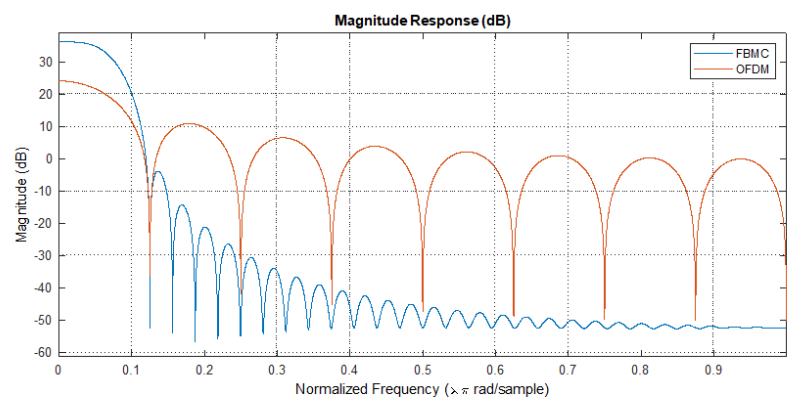

Figure 1: Spectral response comparison between OFDM and FBMC

Like all other Multi carrier modulation schemes (MCM) this approach also suffers from high peak -to - average - power 
ratio (PAPR) and this causes a decrease in the potency of high power amplifier (HPA).To avoid clipping that arises due to the non-linearity of HPA, the amplifiers are made to be operated in linear region with large input back off (IBO), however these amplifiers are driven into saturation resulting in band and out of band distortions. These distortions may lead to high adjacent channel power ratio (ACPR) [4]. The main attractive attribute of FBMC is low ACPR and side lode distortions which are destroyed with the introduction of this clipping. When the PAPR is decreased, the HPA efficiency increase and also ACPR is lowered. This paper focuses on providing a solution to treat and mitigate the high PAPR with ant bee optimization approach.

\section{RELATED WORKS}

Several authors have proposed different approaches to mitigate the PAPR and increase the efficiency of the FBMC systems, few works that are related to the current research work were presented in this section.

Maharaj et.al [5], proposed active constellation extension (ACE) approach for mitigating PAPR in FBMC systems; in this method the authors presented clipping and filtering scheme in addition with ACE and maintained a minimum distance between the constellation points. In this method they observed that PAPR reduction comes at the expense of BER similar to the case in OFDM however, the method outperforms that several overlapping structures that were proposed before this.

Kollar et.al [6] presented an iterative clipping based approach; this approach relies on Buss gang theorem and can be applied at the transmitter side without of generality and properties of the FBMC system. In this paper the authors have proposed a gain metric that is used to determine the optimal number of iterations required to achieve the lowest possible PAPR.

Horvath and others presented a lower bound approach for selecting the optimal and suitable approach. The authors have proposed an optimization approach to attain a significant low PAPR for short FBMC frames of symbols. In this approach the authors have employed both tone reservation and active constellations as the extension of it, thereby achieving a considerable low PAPR. The experimental results depict a low bound PAPR when compared with solo traditional methods however, the complexity has increased making it riskier in selecting the parameters
Skrzypczak et.al [8] proposed, a modified SLM technique that can employ in FBMC system. This technique thereafter termed as OSLM (Orthogonal- SLM)approach. In this technique, a search is performed for longer intervals to capture the overlapping properties of the FBMC system. So, this results in modulating the continuous symbols to achieve the non-overlapping nature of FBMC. In this work, the authors have mainly focused on PAPR problem and proposed a modified SLM approach that finds an optimal solution for low complex FBMC system.

The authors in [9] and [15] have proposed a Sliding Window based Tone reservation (SW-TR) approach. Unlike the method in [8], this method is a clipping based approach that utilizes the full sub carriers to suppress the peaks in the FBMC signal. This approach also employs a sliding window, so that the clipping can be performed in each individual frame of the FMBC signal. Though this technique could able to achieve lower PAPR, this method also suffers from the similar limitations as of Tone reservation of attaining high BER. This rise of BER is due to the addition of non-data carrying sub-carriers due to which a lower throughput is retained causing a rise in Error Rate.

Lu et al [10], uses dynamic programming (DP) based optimization approach for conventional Partial transmit sequence (PTS) method. The DP employed in this work used to find the most optimal phase rotation factors that may yield the lower PAPR of the signal. From the experimental analysis it was observed that this approach is attaining better results than OSLM based FBC approach. However, even this approach also suffers from the limitations like conventional PTS scheme of increase in the computational complexity exponentially with the increase in the proto type filter length and number of phase factors. Ant bee colony optimization approach for the reduction of PAPR in OFDM system was proposed by cheng et. al [11]. In this method they adopted SLM (selective mapping) concept to mitigate the PAPR and shown a good reduction in PAPR with controlled computations. So, from the above discussions it is evident that there is always a scope for new approach that can be used to minimize PAPR in FBMC/OQAM systems.

\section{PAPR ANALYSIS IN FBMC SYSTEMS}

The traditional approaches of PAPR reduction schemes may not be applied for FBMC systems because of its overlapping structure. In order to apply a PAPR reduction approach first one need to know the working model and mathematical analysis of the FBMC system. 
Due to high spectrum efficiency, the FBMC system has drawn much interest in research for developing novel high data rate communication system protocols. In this system the resultant signal is obtaining by adding ' $M$ ', time shifted OFDM/OQAM symbols which are obtained by passing the modulated QAM symbols through prototype filter [12].

The above figure 2 depicts the OFDM/OQAM structure that consists of ' $\mathrm{N}$ ' number of sub -carriers. It was observed that after the symbols undergo QAM modulation they are converted into parallel form which are represented in equation (1)

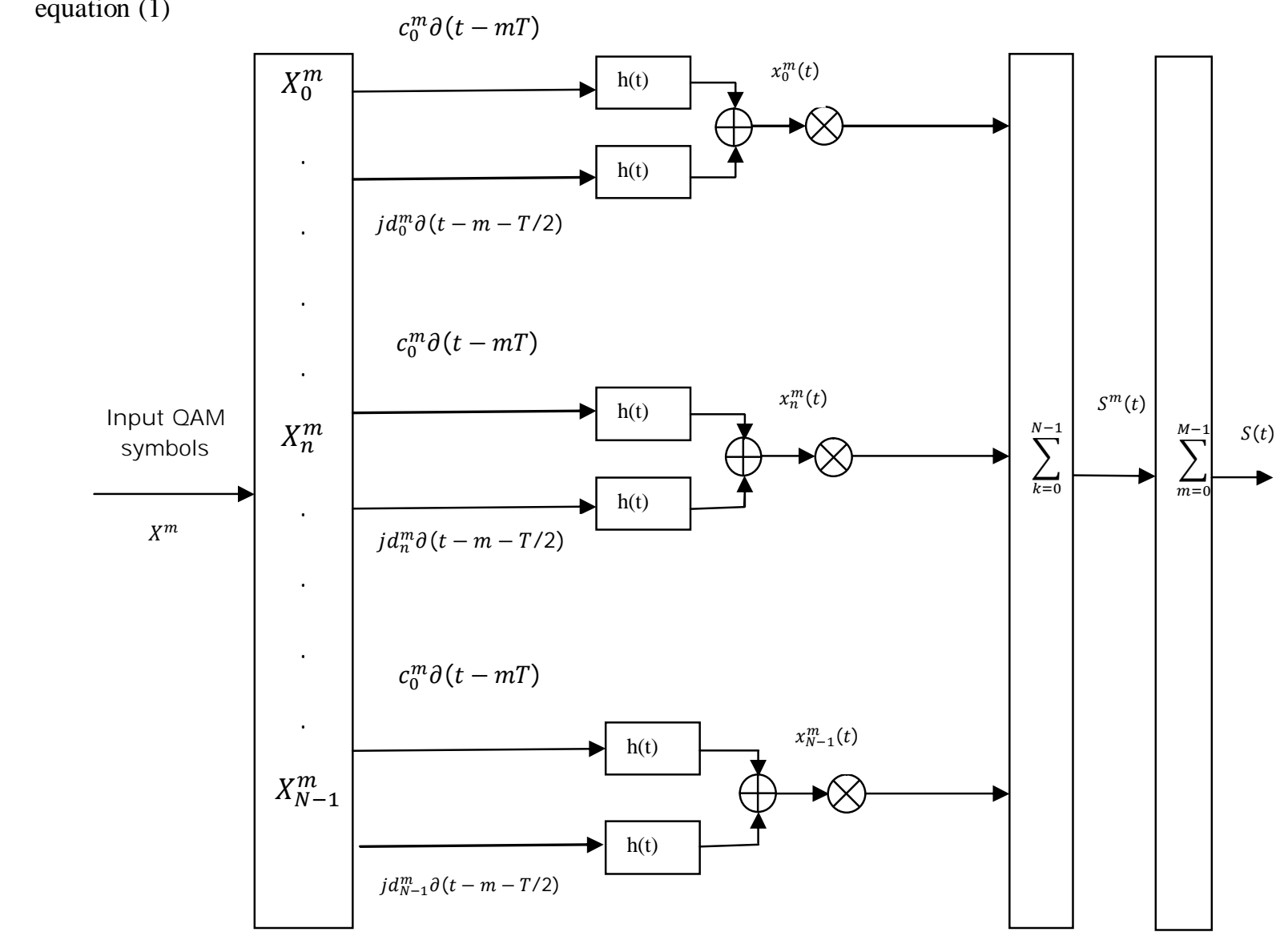

Figure 2: Block diagram of FBMC/OQAM system
Where ' $c$ ' and ' $\mathrm{d}$ ' represents the real and imaginary parts of $X_{n}^{m}$. The real and imaginary parts of $X_{n}^{m}$ are staggered by $\mathrm{T} / 2$ and passed through the prototype filter to obtain

$$
x_{n}^{m}(t)=c_{n}^{m} h(t-m T)+j d_{n}^{m}\left(t-\frac{T}{2}-m T\right)(3)
$$

$X=\left[X^{0}, X^{1}\right.$ .,$\left.X^{M-1}\right](1)$

In the above equation (1) the term ' $M$ ' is the number of data blocks, $\mathrm{X}^{\mathrm{m}}$ is the $\mathrm{m}^{\text {th }}$ data block which is defined as $X^{m}=\left[X_{0}^{m}, X_{1}^{m} \ldots \ldots \ldots . X_{N-1}^{m}\right]^{T}$, Where ' $\mathrm{N}$ ' represents the number of subcarriers then $X_{n}^{m}=c_{n}^{m}+j d_{n}^{m}$
Where $\mathrm{h}(\mathrm{t})$ is the response of the prototype filter. Here in this paper the prototype filter coefficients represented below [13

$$
h(t)=\alpha\left(c(0)+2 \sum_{i=1}^{K-1}(-1)^{i} c(i) \cos \left(\frac{2 i \pi}{K N} t\right)\right)(4)
$$


Where $\alpha$ is the normalization factor and $\mathrm{c}(\mathrm{i}), \mathrm{i}=0,1 \ldots \ldots \mathrm{K}-1$ are given as $\quad \mathrm{c}(0)=1 \quad, \mathrm{c}(1)=0.97195 \quad \mathrm{c}(2)=0.7071$ $c(3)=0.23514$

The modulated symbols in $\mathrm{N}$ orthogonal sub carriers is represented as

$$
S_{n}^{m}(t)=\left\{c_{n}^{m} h(t-m T)+j d_{n}^{m}\left(t-\frac{T}{2}-m T\right)\right\} e^{j n\left(\frac{2 \pi}{T} t+\frac{\pi}{1}\right)}(5)
$$

For $\mathrm{K}=0,1,2 \ldots \mathrm{N}-1$. Then $S_{n}^{m}(t)$ on all the $\mathrm{N}$ sub carriers are added up together to obtain

$$
S^{m}(t)=\sum_{n=0}^{N-1} s_{n}^{m}(t)
$$

The desired FBMC/OQAM signal is represented as

$$
s(t)=\sum_{m=0}^{M-1} S^{m}(t)
$$

For $0 \leq t \leq\left(M+N-\frac{1}{2}\right) T$. Finally, the PAPR for the system is represented as

$$
P A P R=10 \log 10\left(\frac{\max _{p T \leq t \leq\left(p+\frac{1}{2}\right) T^{|l(t)|^{2}}}}{\text { Pave }}\right)
$$

Where Pave: is the average power of $\mathrm{S}(\mathrm{t})$. The spectral magnitude response of FBMC system is depicted below in figure 3 , it can be observed thatas the $\mathrm{K}$ value increases the side lobe response is considerable negotiable and very minute; this is the advantage of FBMC for large number of sub carriers.

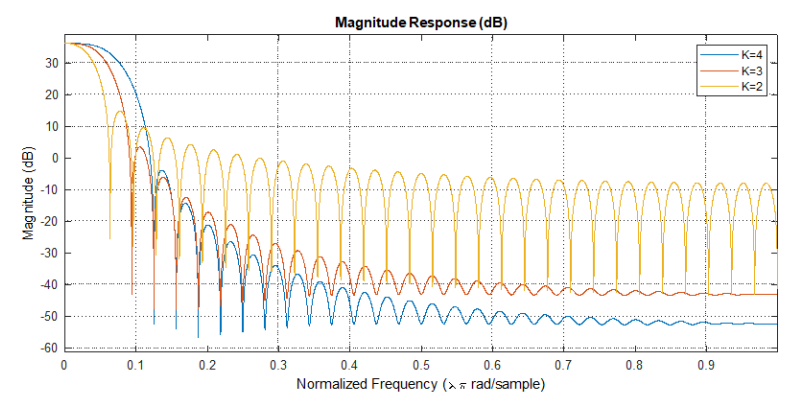

Figure 3: Spectral magnitude response FBMC at different $\mathrm{K}$ values

\section{PROPOSED WORK}

The block diagram of the proposed approach is depicted in figure 4.

The output obtained with equation (7) is processed for IFFT operation, thus obtained symbols are convolved with phase factors and the one sub carrier which attains low PAPR is selected. However, in our approach the phase optimization is performed with $\mathrm{ABC}$ algorithm.

On other hand, the Ant Bee Colony (ABC) is a swarm based optimization approach that mainly operates similar to the behavior of honey bees. This algorithm mainly consists of three phases namely, employed bees, scout bees and onlooker bees. In this algorithm the position of the nectar and the amount of food available represent a possible solution. This solution makes us to help in find the optimal phase factor $\mathrm{P}^{\mathrm{u}}$. This optimal phase factor and fitness value is the key for obtaining low PAPR. So to retain the best rotation phase factor is to find the optimal food source that has maximum fitness value.

At first, the phase factors are randomly generated $\mathrm{P}^{\mathrm{u}}$ and the fitness value of each factor is calculated using the equation (10)

$$
f i t\left(P_{i}^{U}\right)=\frac{1}{1+f\left(P_{i}^{u}\right)}
$$

From the above equation (1) it is evident that lower PAPR can be achieved with higher fitness value function. Employed bees move for new food sources aiming to find the best qualified sources within the neighborhood of the present source which is given by equation (11)

$$
P_{i}^{u \prime}=P_{i}^{u}+\emptyset_{i}\left(P_{i}^{u}-P_{k}^{u}\right)
$$

In the above equation (11) $P_{k}^{u}$ is an optimal solution obtained within the neighborhood of $P_{i}^{u}$ and $\emptyset_{i}$ is a random number in the range $[-1,1]$. The updated fitness value $P_{i}^{u \prime}$ is calculated by equation (10) (11) and in case when $f i t\left(P_{i}^{u \prime}\right)>\operatorname{fit}\left(P_{i}^{u}\right)$ then the bee marks the current position as new position $\left(P_{i}^{u \prime}\right)$ and discards the earlier position $\left(P_{i}^{u}\right)$. When all the bees complete the search process, the nectar is accumulated and the position information is updated to onlooker bees. These onlooker bees move to new position for food which can be represented mathematically as in equation (12) . 


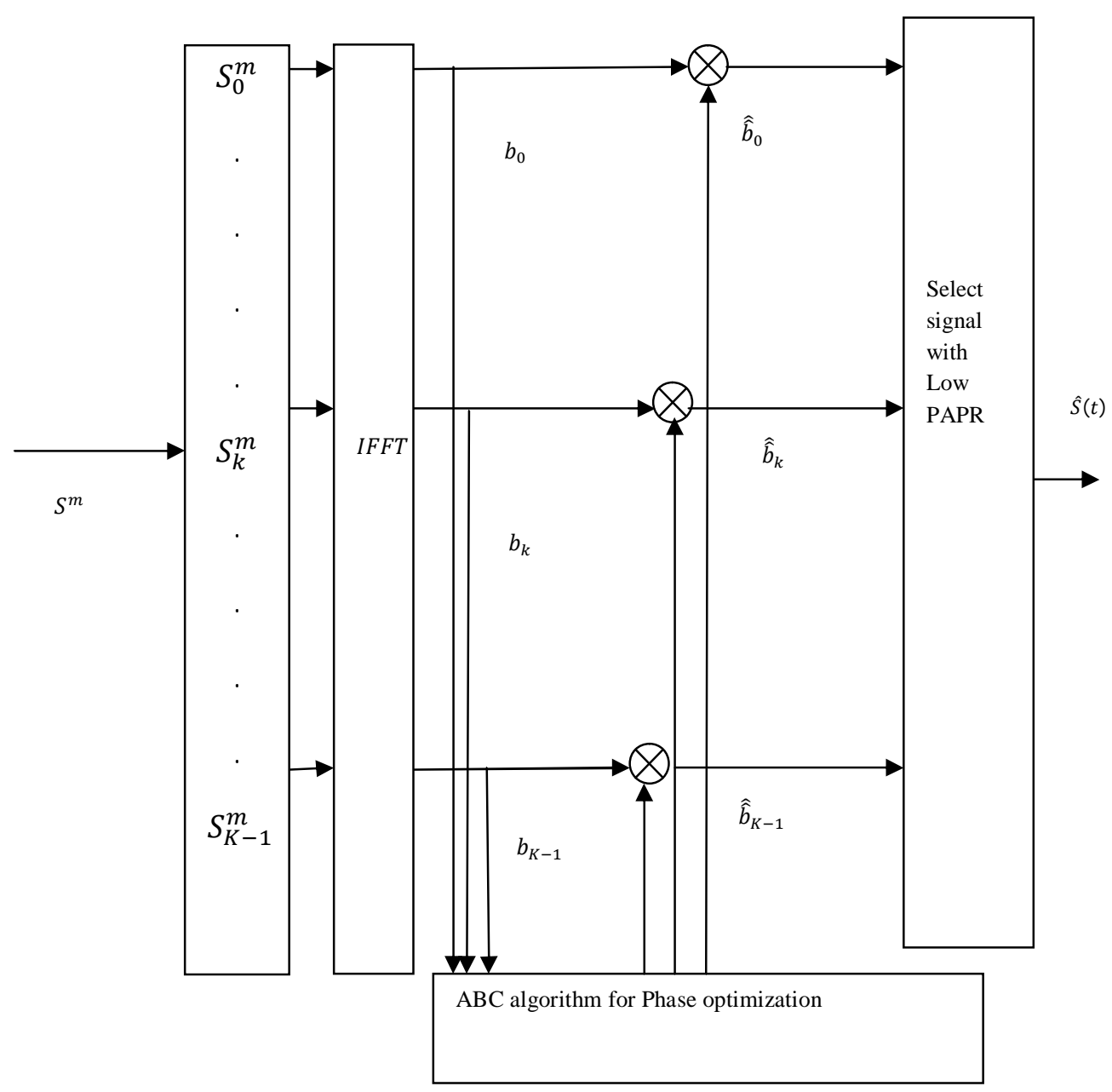

Figure 4: Proposed ABC based phase optimization approach for FBMC

$$
p_{i}=\frac{f i t\left(P_{i}^{u}\right)}{\sum_{i=1}^{S N} f i t\left(P_{i}^{u}\right)}
$$

In the above equation (12) the term ' $\mathrm{SN}$ ' is the population size which is total number of employed and onlooker bees. When the fitness value is not improved within the limit value even after the employed and onlooker bees completing the job then the scout bees look for new sources which here can be treated for optimal rotation phase factor from randomly generated factors. This can be represented mathematically as shown in equation (13).

$$
P_{i}^{u \prime}=\min \left(P_{i}^{u}\right)+\operatorname{rand}(1) \cdot\left(\max \left(P_{i}^{u}\right)-\min \left(P_{i}^{u}\right)\right)
$$

\section{EXPERIMENTAL RESULTS}

The performance assessment is conducted with $\mathrm{N}=512$ sub carriers with 4,16 QAM modulation schemes. The proposed approach is evaluated and compared against the methods discussed as FBMC SLM [8] [14]. CCDF (Complementary cumulative distribution Function), PSD (Power spectral density) are the parameters that were used to evaluate the performances. 


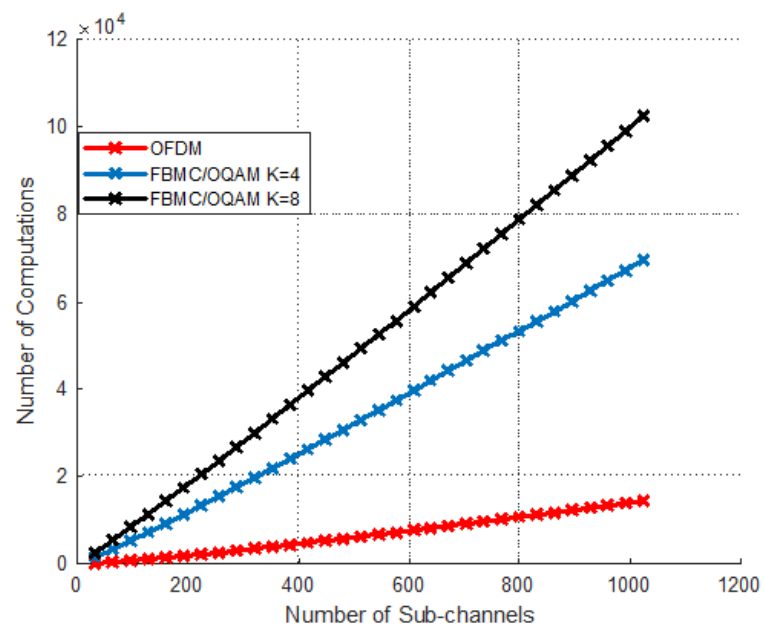

Figure 5: Computational complexity comparison for OFDM and FBMC at different $\mathrm{K}$ values

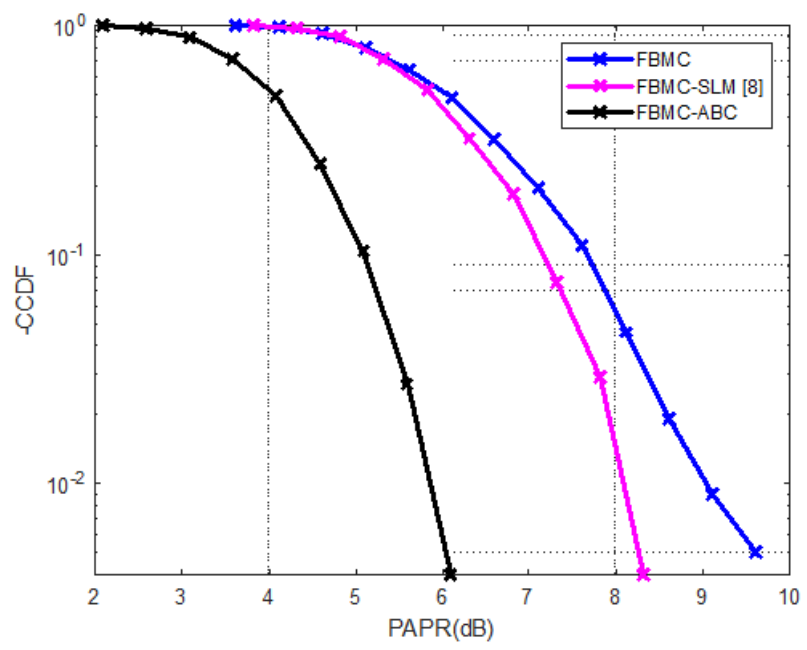

Figure 6: CCDF Vs PAPR for the proposed approach and its comparison with FBMC -SLM [8], N=512, 4-QAM modulation

The analysis is made with different varying parameters like number of sub carriers, modulation schemes. It can be observed from the experimental results that the proposed approach could able to reduce the PAPR by $3.8 \mathrm{~dB}$ on average when compared with original FBMC PAPR and $2.2 \mathrm{~dB}$ when compared against the traditional FBMC-SLM approach which is quite significant achievement. This is slightly increased by $0.8 \mathrm{~dB}$ when the number of sub carriers are increased from 512 to 1024 , however the approach outperforms than the existing approaches.

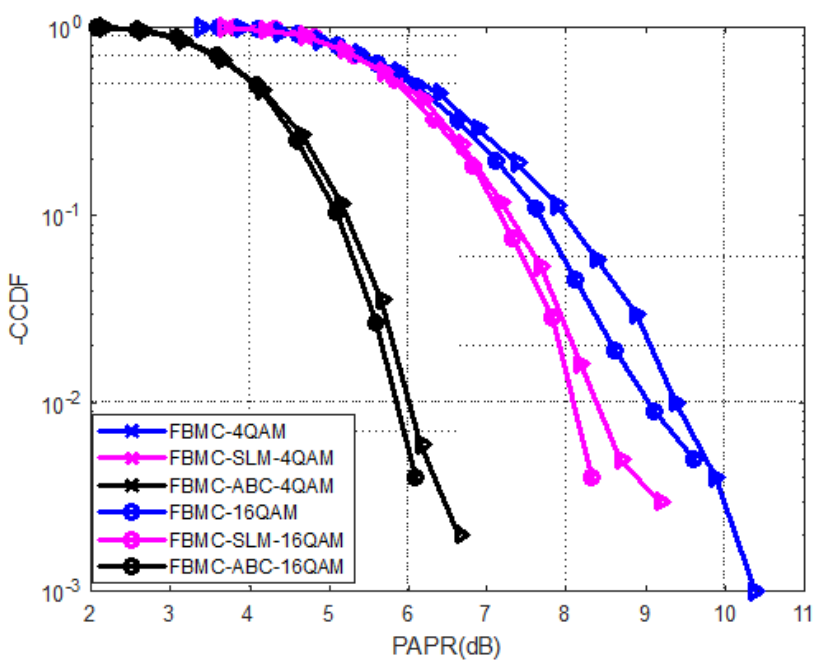

Figure 7: CCDF Vs PAPR for the proposed approach and its comparison with FBMC-SLM for 4,16 QAM

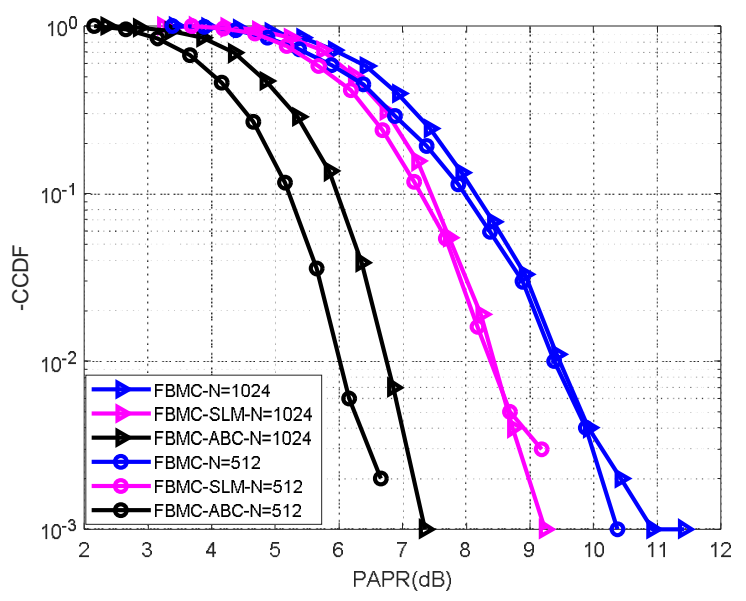

Figure 8: CCDF Vs PAPR for the proposed approach and its comparison with FBMC-SLM for N=512,1024 with 16QAM modulation

\section{CONCLUSIONS}

This paper proposes an optimization approach for phase factors in conventional SLM method that is applied to FBMC system. The problem of optimization is solved with the help of ABC algorithm with which the PAPR could be able to decrease by $2 \sim 2.3 \mathrm{~dB}$ on average when compared against the original FBMC PAPR. However, the method computations are higher than the traditional approaches which consumes more time for processing than earlier methods. From above all discussions it can be concluded that this method able to achieve the objective of the work by mitigating the PAPR for considerable amount and thereby can be used for high performance FBMC. 


\section{REFERENCES}

[1] R.K. Jain, SumitKatiyar, NK.Agarwal,"Survey of latest wireless cellular Technologies for Enhancement Of Spectral density at reduced cost”, IJCSI, Vol.8, issue 3, No.2, May 2011.

[2] B. Saltzberg, "Performance of an efficient parallel data transmission system", IEEE Transactions on Communication Technology, 15-6, 805 to 811, Dec. 1967

[3] M. Bellanger, "Physical layer for future broadband radio systems," 2010 Radio and Wireless Symposium Conference [4] T. Jiang, C. Li and C. Ni, "Effect of PAPR Reduction on Spectrum and Energy Efficiencies in OFDM Systems With Class-A HPA Over AWGN Channel," in IEEE Transactions on Broadcasting, vol. 59, no. 3, pp. 513-519, Sept. 2013.

[5] N van der Neut, B Maharaj, F de Lange, G Gonzalez, F Gregorio, J Cousseau, in EURASIP Journal on Advances in Signal Processing. "PAPR reduction in FBMC using an ACE-based linear programming optimization", vol. 2014, no. 172 ,Springer Publications, 2014

[6] Z Kollar, L Varga, B Horvath, P Bakki, J Bito, in Hindawi Scientific World Journal. Evaluation of Clipping Based Iterative PAPR Reduction Techniques for FBMC Systems, vol. 2014, no. 841680,Hindawi Publications, 2014 [7] Horvath, Balint\& Horvath, P, "Establishing lower bounds on the peak-to-average-power ratio in Filter bank multicarrier systems". 7. 10-16, 2015

[8] A. Skrzypczak, J. Javaudin, and P. Sinhan, "Reduction of the peak-to average power ratio for the OFDM/OQAM modulation," in Proc. IEEE VTC, Melbourne, Vic., Australia, May 2006, pp. 2018-2022

[9] S. Lu, D. Qu, and Y. He, "Sliding window tone reservation technique for the peak-to-average power ratio reduction of FBMC-OQAM signals," IEEE Wireless Commun. Lett., vol. 1, no. 4, pp. 268271, Aug. 2012.

[10] D.Qu,S.Lu and T.Jiang, “ Multi block joint optimization for the peak to average power ratio reduction of FBMC-OFDM signals", IEEE transactions on signal processing, Vol 61, no7, Pp-1605-1613,Apr-2013

[11] X. Cheng, D. Liu, S. Feng, H. Fang and D. Liu, "An artificial bee colony-based SLM scheme for PAPR reduction in OFDM systems," 2017 2nd IEEE International Conference on Computational Intelligence and Applications (ICCIA), Beijing, 2017, pp. 449-453.

[12] Chen, D. Qu, and T. Jiang, "Prototype filter optimization to minimize stop band energy with NPR constraint for filter bank multicarrier modulation systems," IEEE Trans. Signal Process., vol. 61, no. 1, pp. 159-169, Jan. 2013.

[13]PHYDYAS Project-D5-1.http://www.ictphydyas.org/delivrables/PHYDYAS-D5-1.pdf

[14] B.Kedarnath, Dr. Shah Aqeel, Dr. Syed Abdul Sattar, "BER Analysis and PAPR Reduction of FBMC/OQAM Systems", IJAREC, Vol- 6, Issue-12, December-2017

[15] V. S. Kumar and S. Anuradha, "Sliding window tone reservation using smart gradient projection method for PAPR reduction of FBMC signals," in Proc. Int. Conf.
Elect., Electron., Signals, Commun. Optim., Jan. 2015, pp. 494499. 